\title{
GNSS permanent stations control by the means of local ties monitoring
}

\author{
Karolina Szafranek ${ }^{\mathrm{a}}$, Stanisław Schillak ${ }^{\mathrm{b}}$, Andrzej Araszkiewicz ${ }^{\mathrm{a}}$, \\ Mariusz Figurski ${ }^{a}$, Marek Lehmann ${ }^{b}$, Paweł Lejba ${ }^{b}$ \\ ${ }^{a}$ Faculty of Civil Engineering and Geodesy, Military University of Technology, Kaliskiego 2, Warsaw 00-908, Poland \\ ${ }^{b}$ Astrogeodynamic Observatory, Space Research Centre, Polish Academy of Sciences, Drapałka 4 62-035 Kórnik, Borowiec, Poland
}

\begin{abstract}
The paper presents the method of GNSS (Global Navigation satellite System) permanent stations control by the monitoring of local ties between GNSS and SLR (Satellite Laser Ranging) stations. Co-locations are defined as two or more instruments occupying very close locations, which are very precisely surveyed by ground measurements in all three dimensions. The vector determined by classical or satellite methods is called a local tie. Every change of stations position obviously results in change of local tie value. The comparison of two independent stations' coordinates enable to distinguish the real movement of one of the stations, potential processing errors and equipment malfunction.

The analysis were based on the data from several globally distributed GNSS-SLR sites. The data gathered between 1996-2012 by these two techniques were processed using coherent strategies (the same models and parameters were used). Monthly orbital arcs for laser observations were created basing on solutions from several SLR stations with the best solutions quality and the highest amount of observations. For GNSS stations coordinates determination about 130 sites belonging to the IGS (International GNSS Service) were selected: 30 with local ties to SLR sites and others basing on their geolocalization and solutions time series analysis. The GNSS and SLR station coordinates were expressed in ITRF2008 for the epoch of 2005. The GNSS solutions were reduced to the SLR markers positions using local ties values and then expressed in topocentric frame $N E U$ for better interpretation.

The analysis of differences between SLR and GNSS NEU coordinates proved that mutual control of two techniques can be helpful in detection of problems at specific GNSS stations like e.g. equipment failure or improper GNSS antenna's calibration. Comparisons of different techniques results enable to distinguish stations specific causes of coordinates discontinuities and phenomena with wider range like earthquakes. In case of temporal disturbances the proper actions can be taken, but the permanent change of the coordinates force the necessary of another local tie determination, because of the importance of inter technique connection for global reference frame construction.
\end{abstract}

Keywords: GNSS; reference station; SLR; local tie; monitoring.

\section{Introduction}

GNSS (Global Navigation Satellite System) permanent stations are widely used for reference system realization, therefore every change of their position should be reflected in the update of their coordinates, because it has an impact on the accuracy of differential measurements. Besides exact localization, they serve as the reliable and dense input for geodynamical models, as their velocities are determined on the basis of the long periods of observations. The paper describes the method of GNSS permanent stations monitoring by the means of comparison between the GNSS and SLR (Satellite Laser Ranging) stations coordinates. This method can strengthen and supplement the monitoring of coordinates with reference to their most reliable values (e.g. mean values calculated on the basis of long-term observations or cumulative solution [1]). The joint processing of various techniques is currently very up-to-date subject. Analysis of GNSS and SLR observations performed for TRF determination described e.g. in [2] but they concerned mainly SLR observations of GNSS satellites equipped with laser reflectors.

Co-locations are defined as two or more instruments occupying very close locations, which were very precisely surveyed by ground measurements in all three dimensions. The vector determined by classical or satellite methods is called a local tie. The local ties have a very significant role in construction of global reference frame e.g. ITRF2008 [3], so there is a very

Corresponding author: Karolina Szafranek. E-mail address: kszafranek@wat.edu.pl

http://dx.doi.org/10.3846/enviro.2014.248

(C) 2014 The Authors. Published by VGTU Press. This is an open-access article distributed under the terms of the Creative Commons Attribution License, which permits unrestricted use, distribution, and reproduction in any medium, provided the original author and source are credited. 
strong need to determine them with the highest possible accuracy. Every change of stations position obviously results in the change of local tie value.

The main problem concerning the coordinates monitoring is the interpretation of the reasons of their changes. They can be related to the hardware malfunction, errors in the processing, some external phenomena like earthquakes and many others. The most important matter is to distinguish the real movement of the stations and changes of the coordinates caused by other factors usually strictly connected to the one technique. One of the best method is to compare the results (coordinates) of two independent stations. Use of stations, which gather the different kind of data (like GNSS and SLR), can be especially fruitful here.

\section{Methodology}

The GNSS and SLR data gathered by selected IGS (International GNSS Service, [9]) and ILRS (Internationa Laser Ranging Service, [10]) stations were processed separately using consecutively Bernese 5.2 [5] and Geodyn-II [6] software. The main goal of the processing was to obtain the result (coordinates and velocities) using the coherent processing strategy in terms of using the same parameters and models (in agreement with the [7]) and to verify their agreement. According to the GGOS (Global Geodetic Observing System, [8]) requirements, the accuracy of measurements made using global techniques should not exceed 1 centimetre. Strategies of SLR and GNSS processing were described in details in [4]. For analysis data gathered by selected IGS and SLR stations in the period 1996-2012 were used. Only LAGEOS-1 and LAGEOS-2 data were used to determine SLR coordinates. Monthly orbital arcs for laser observations were created using solutions from several SLR stations with the best solutions quality and the highest amount of observations. It led to the SLR solutions with temporal resolution of 1 month. Strategy of GNSS processing was in accordance with IGS and EUREF recommendations. The main problem concerned processing of the global network for the data gathered between 1996-2000 because of small number of stations. It caused very long baselines and made determination of ambiguities impossible. Because of that, the whole global network was divided into 7 smaller subnetworks with continental range. The subnetworks were processed separately. At the end they were combined together using ADDNEQ2 module of Bernese software.

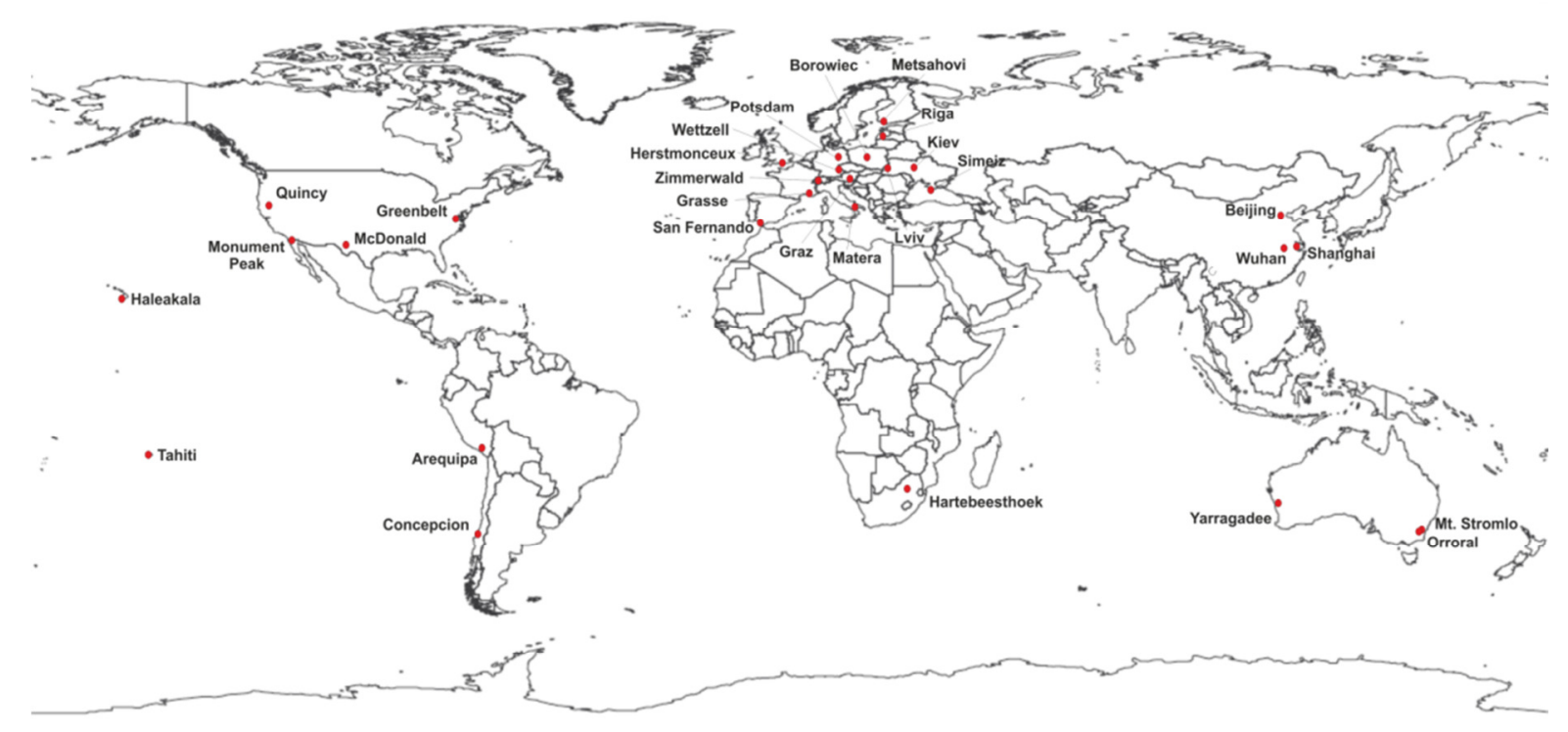

Fig. 1. Global distribution of the stations with GNSS and SLR sites related using local ties (stations analyzed in the frame of local ties monitoring) [4]

The GNSS and SLR station coordinates were expressed in orto-Cartesian, geocentric $X Y Z$ coordinates (ITRF2008 for the epoch of 2005). The GNSS solutions were reduced to the SLR markers positions using local ties values and then expressed in topocentric frame $N E U$ for better interpretation. The local ties values were taken from ITRF2008 or, in some cases, from other sources (e.g. reports from measurements conducted for the local tie determination). The analysis and comparisons were performed for GNSS and SLR NEU time series for each collocation station (Fig. 1). They were performed mainly to compare the quality of the both technique results, but it clearly appeared that such comparison is a very good method to notice different kinds of problems appearing at GNSS and SLR stations and to estimate their importance.

\section{GNSS stations monitoring by comparison with SLR}

Analysis were conducted on the basis of GNSS and SLR NEU time series for each collocation station. Local ties are defined as two or more stations (co-location stations) gathering data using various techniques, which mutual position was determined very precisely using classical or GNSS method. Topocentric $N E U$ values were calculated with the reference to the SLR marker and its coordinates expressed in the ITRF2008. The assumption is that both GNSS and SLR stations should 
give the same results if there is no external problem affecting the stations activity and the equipment works correctly (as the strategy of both type of data was coherent). Comparing two types of data allows to verify them and to distinguish the real movement of the stations and changes of the coordinates caused by other factors usually strictly connected to the one technique. Comparing coordinates in near real time could help to avoid long-term equipment problems and take the proper action to eliminate the reason of the problem. It is a common fact that GNSS plays a control role for the SLR station, as the GNSS technique is much cheaper and, as a consequence more common, but the verification of the data correctness is of course mutual.

When analyzing the $N E U$ time series, the systematic error of local tie measurement has to be taken into consideration. It is assumed that accuracy of local ties measurements is unlikely to be less than $3 \mathrm{~mm}$, mainly because of the difficulties with the reliable and precise reference point determination. In practice, many of the differences between ground measurements and values calculated by each technique data processing turn out to be significantly larger. Such error can be clearly seen in $N E U$ time series as the systematic shift between both types of results. Monitoring of the local ties values is the important issue, especially for the preparation of the next ITRF (ITRF2013), but it is not a subject of this paper. The goal of this paper is to show that investigation of co-location stations is a great method of controlling both GNSS and SLR coordinates.
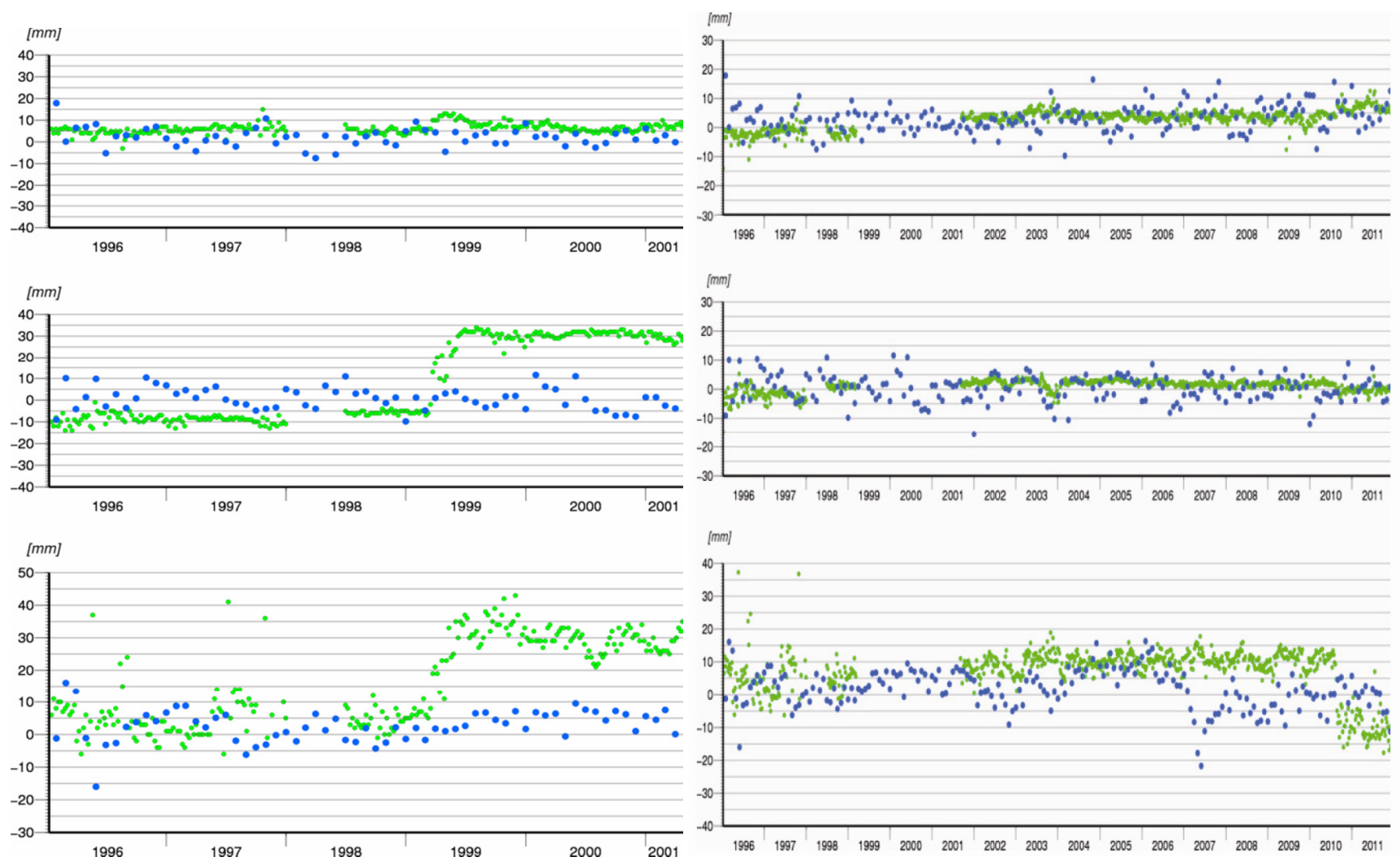

Fig. 2. Time series of NEU components of the GNSS station HERS (green) and SLR station 7840 (blue). On the left (a) the coordinates change resulted from the antenna failure can be observed. On the right (b) time series without unreliable values are presented

One of the best examples of the necessity of mutual control is site consisting of GNSS station HERS and SLR station 7840 (Herstmonceux, United Kingdom). In the first half of 1999 a significant change of GNSS coordinates (30 millimetres for East and $U p$ components) can be observed, while SLR results did not change (Fig. 2a). According to the [11], the reason of coordinates change was antenna failure - signals reception was dependent on its azimuth orientation. This defect was eliminated in September 2001 [12], so it caused more than two years of gap in the HERS data series. Comparing the GNSS and SLR data gives the exact answer about the reason of such effect - it could not be related to any external disturbance, as it would had an impact both on GNSS and SLR positions. In the second half of 2010 anther discontinuity in GNSS coordinates can be observed. This time it is probably caused by the change of the antenna in September. Such discontinuity should be followed by another local tie determination mainly for the next ITRF construction purpose.

The possibility of mutual control of GNSS coordinates increases with the number of co-located instruments working in the same time. Figure 3a presents time series of NEU coordinates of three different instruments: GNSS GRAS station (green) and two SLR stations: 7835 (navy blue) and 7845 (blue) in Grasse, France. Unfortunately, SLR stations did not have common period of measurements, but both of them can be used as a control for the GNSS one. Up to 2001 the good agreement in all components between both time series can be observed. In 2001 about 25 millimetres difference occurred between GRAS and 7835 Up components, but as the discontinuity concern SLR coordinates it is more likely that this effect is caused by some changes at SLR station. The new SLR station 7845 launched in 2008 shows the very good agreement in all three components with the GNSS coordinates. 
The GNSS-SLR site in Yaragadee (Australia) consists currently of GNSS YAR2 (green) and 7090 SLR stations (Fig. 3b). Up to 1998 the second GNSS station YAR1 was in operation (grey), so in that time coordinates of two GNSS (GPS only on that time) stations can be compared. They were not totally separate instruments, as they shared the same antenna, so the reference point was the same for both YAR1 and YAR2 (they had different receivers). Anyway, it does not prevent from some differences in coordinates. The agreement in horizontal components between YAR2 and 7090 is very good for the whole time of observations, but there are some differences concerning Up component especially before 2002. Both types of time series show oscillations, but they can be more clearly seen in GNSS solutions because of the smaller discrepancy.
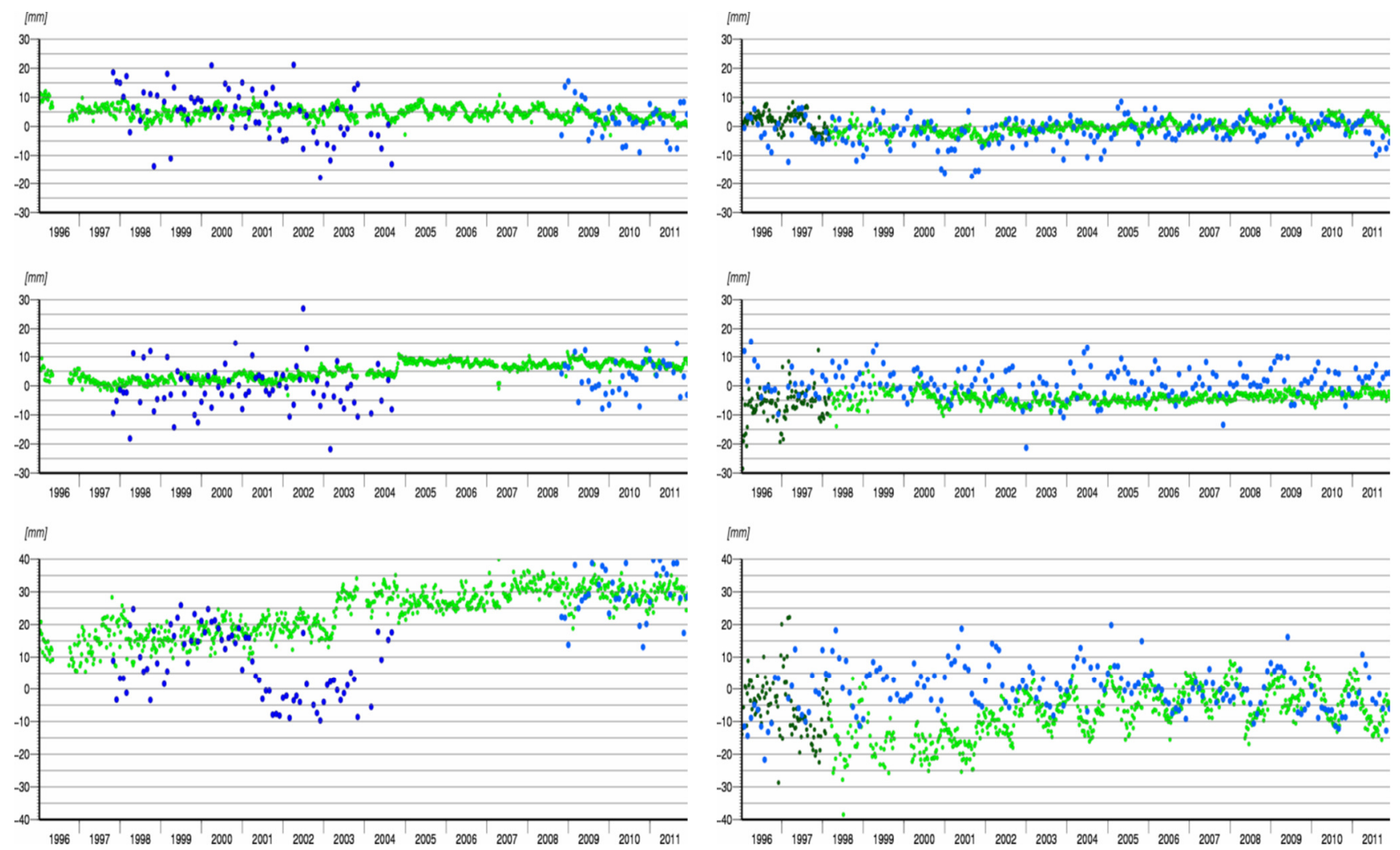

Fig. 3. On the left (a) - time series of NEU components of the GNSS station GRAS (green) and SLR stations 7835 (navy blue) and 7845 (blue). On the right (b) time series of the YAR1 (grey), YAR2 (green) and 7090 (blue) site

Another example of multi-station is at the Australian Capital Territory. The distance between 7843 and 7825 is about 36 kilometres. Time series of 7843, 7849 and 7825 were presented both in the Fig. 4a and Fig. 4b (consequently marked with grey, navy blue and blue). Figure 4a presents also GNSS STR1, while Fig. 4b GNSS TIDB solutions. They were not presented on the same figure, as there is no local tie measured between STR1 and TIDB. The agreement between GNSS and all SLR stations is very good except for the Up component of 7843 SLR station. The disagreement can be observed when compare 7843 topocentric coordinates with all four other stations, so it rather proves the imperfection of the local tie for this station. The gap in 2003 was caused by a serious Canberra fires, which harmed Mount Stromlo site. It explains some change in Up component of the STR1 station. The 7825 replaced 7849, but when comparing its coordinates to the TDB station (not harmed by the fire) it is clearly seen that then new instrument was placed in the exact position of 7849 .

As it was mentioned before, the control between SLR and GNSS is mutual, so GNSS time series can be a base for detection of some internal problems affecting SLR station. The example is given in Fig. 5a for the station in Wettzell (Germany). Both horizontal components of GNSS WTZR and SLR 8834 are in accordance, while some disturbances can be noticed in SLR time series of Up component in 2009-2010. Changes of the resulted coordinates reach the value of 40 millimetres, when there is no alarming change occurring in GNSS time series in that time. The coordinates stabilized after two years in 2011 .

The special care should be put on the stations affected by earthquakes. They can caused some serious discontinuities, not only in coordinates time series, but also in stations velocities. Depending on the strength of the earthquake, time when station stabilize its position can take even up to a few years. When two instruments are placed near to each other the change in their coordinates should be comparable. Figure 5 b presents time series of Monument Peak site (USA) GNSS MONP and SLR 7110 stations. The earthquake took place in 2010 causing 20 millimetres change of the East GNSS component. It can be clearly seen that position of this station was changing until at least 2012 (analysis stopped here). Similar, but less visible, because of the higher scatter, effect can be observed for SLR East component. There is also some stronger linear trend in MONP Up component (probably also related with the earthquake), but it cannot be seen in 7110 vertical coordinates. This should be investigated using also the data from 2012 and 2013. 

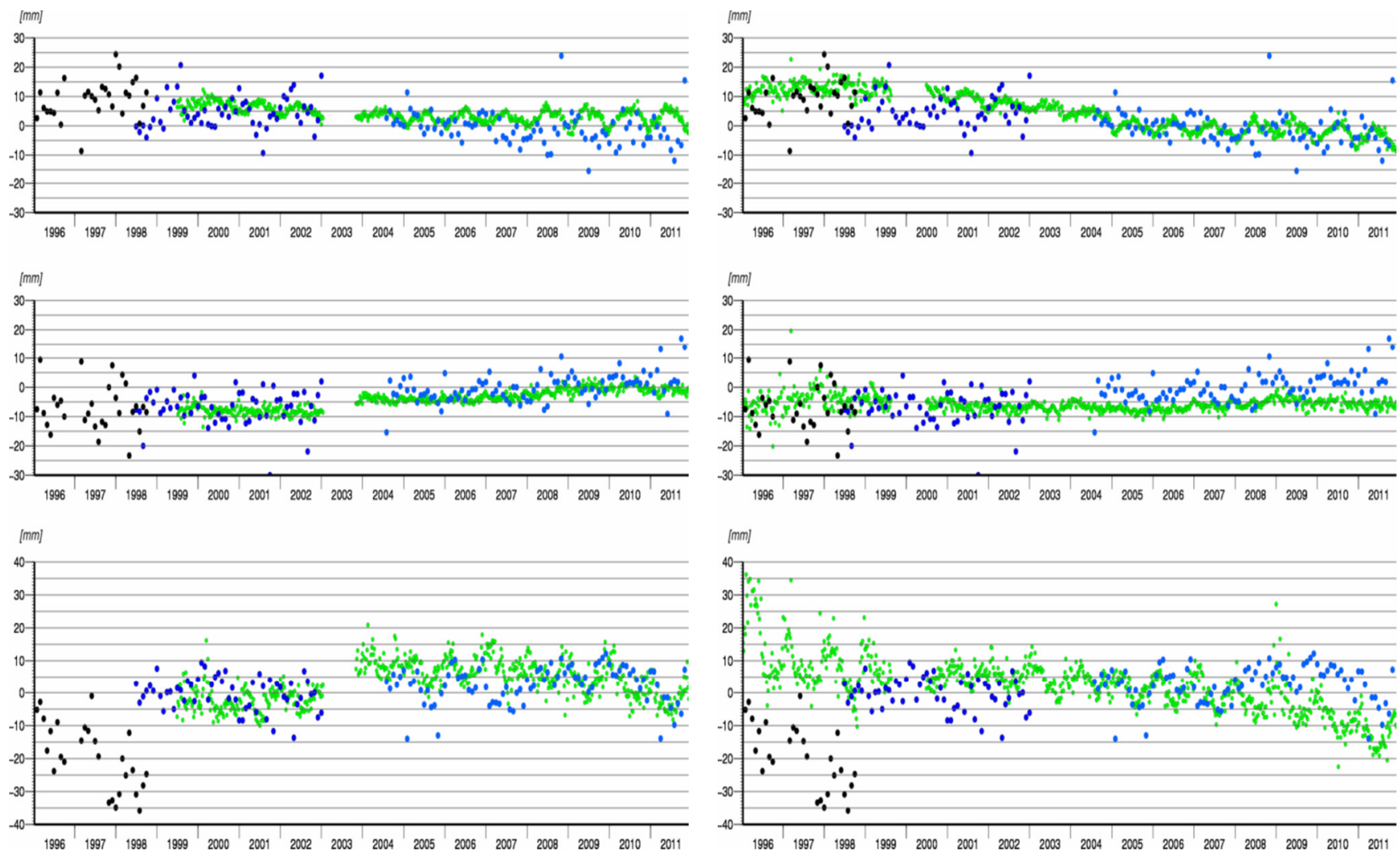

Fig. 4. On the left (a) - time series of NEU components of the GNSS station STR1 (green) and SLR stations 7843 (grey), 7849 (navy blue) and 7825 (blue). On the right (b) time series of the TIDB (green) and SLR stations 7843 (grey), 7849 (navy blue) and 7825 (blue)
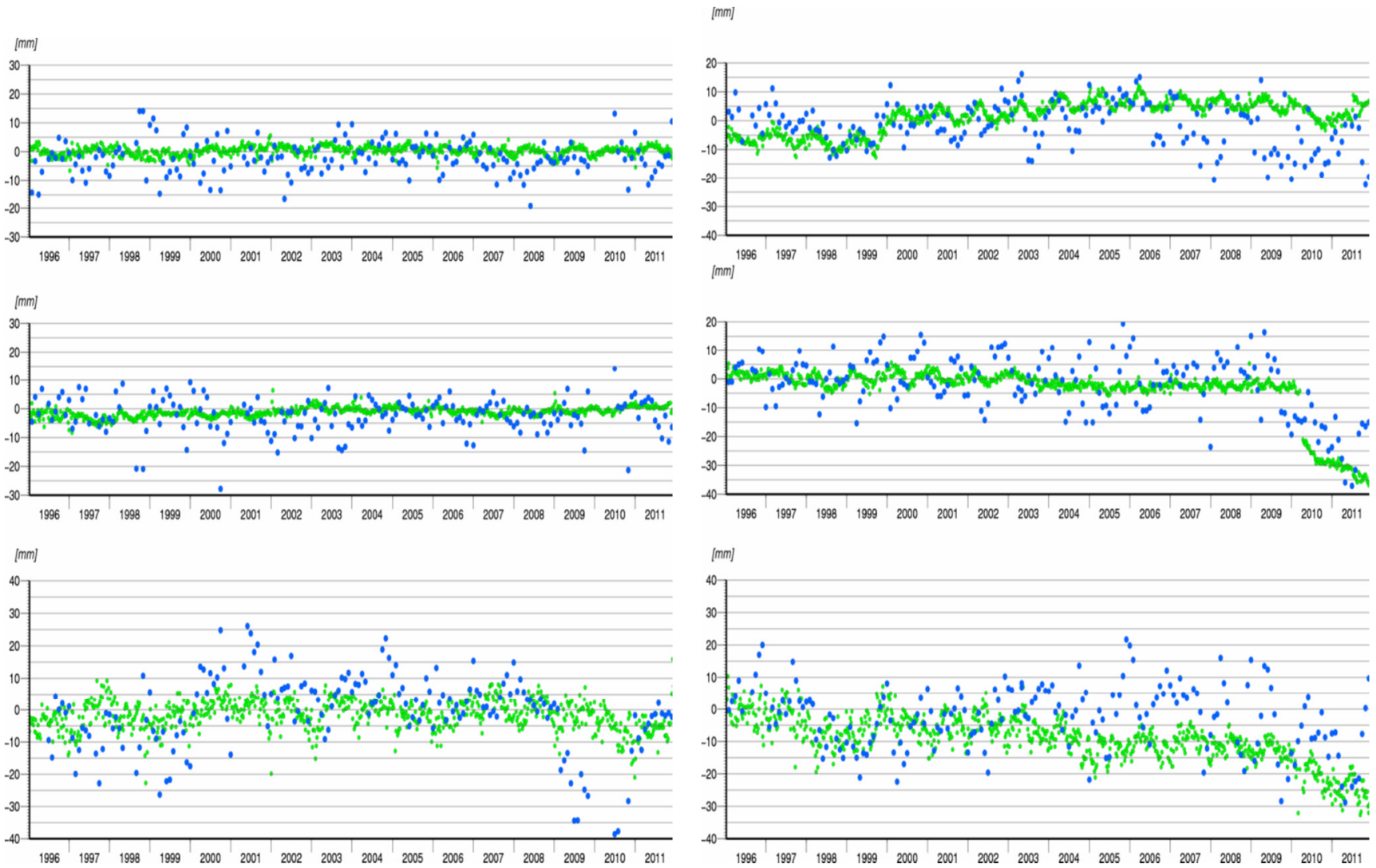

Fig. 5. On the left (a) - time series of NEU components of the GNSS station WTZR (green) and SLR station 8834 (blue). On the right (b) time series of the MONP (green) and SLR station 7110 (blue) 


\section{Conclusions}

The analysis of the SLR and GNSS position time series proves that stations located in close area can play a role of mutual control of coordinates. The goal of the paper was to show the possibility of GNSS monitoring using other techniques, but generally it concerns all types of instruments working as one site (one observatory). GNSS technique can be even more appropriate for this purpose in some cases, because of smaller discrepancies of the solutions and usually higher temporal resolution of obtained solutions. On the other hand, coordinates determined using GNSS technique are strongly related to the processing process and differential approach (if applied), so other source of information is helpful to verify its solutions.

The analysis of coordinates determined using data from two different techniques helps in detection of problems at specific stations like e.g. equipment failure or improper GNSS antenna's calibration. In general, comparisons of different techniques results enable to distinguish between stations specific causes of coordinates discontinuities (HERS-7840 example) and phenomena with wider range like earthquakes (MONP-7110 example). In case of temporal disturbances are noticed, the proper actions can be taken. The faster the reaction is, the smaller amount of data is lost. The permanent change of the coordinates should be followed by another local tie determination. The analysis described in the paper gave the examples how to distinguish the real movement of the stations and changes of the coordinates caused by other factors usually strictly related to the one particular technique.

As the GNSS technique is relatively cheap, GNSS receivers should be always installed near other techniques instruments (like SLR or VLBI), especially if they are of high importance e.g. they are used for the ITRF determination. The analysis of time series can also be used to estimate the quality of local ties, which are crucial for the global reference frame maintenance.

\section{Acknowledgements}

The authors thank SOPAC for GPS Time Series, NASA GSFC for consent to use GEODYN-II program and ILRS and IGS services for their continuous efforts to provide high-quality SLR and GPS data. The research was supported by the grant No. N N526 159540 (,Analysis of differences between sites' coordinates determined using SLR and GPS techniques performed in the frame of Global Geodetic Observing System (GGOS) realization") of the National Science Centre (Poland).

\section{References}

[1] Szafranek, K.; Bogusz, J.; Figurski, M. 2013. GNSS reference solution for permanent station stability monitoring and geodynamical investigations: the ASG-EUPOS case study, Acta Geodynamica et Geomaterialia 10(1/169): 67-75.

[2] Thaller, D.; Dach, R.; Seitz, M.; Beutler, G.; Mareyen, M.; Richter, B. 2011. Combination of GNSS and SLR observations using satellite co-locations, Journal of Geodesy 85(5): 257-272. http://dx.doi.org/10.1007/s00190-010-0433-z

[3] Altamimi, Z.; Collilieux, X.; Métivier, L. 2011. ITRF2008: an improved solution of the International Terrestrial Reference Frame, Journal of Geodesy 85(8): 457-473. http://dx.doi.org/10.1007/s00190-011-0444-4

[4] Szafranek, K.; Schillak, S. 2013. Introduction to joint analysis of SLR and GNSS data, Reports on Geodesy and Geoinformatics (formerly Reports on Geodesy) 1(92): 143-154.

[5] Dach, R., U. Hugentobler, P. Fridez, M. Meindl (Eds), 2007. Bernese GPS Software Version 5.0. User manual, Astronomical Institute, University of Bern.

[6] Pavlis, D. E.; Luo, S.; Dahiroc, P. et al. 1998. GEODYN II System Description. Hughes STX Contractor Report, Greenbelt, Maryland.

[7] Petit, G.; Luzum, B. (Eds.). 2010. IERS Conventions (2010), IERS Technical Note No. 36.

[8] Plag, H. P., Pearlman, M.(Eds.). 2009. The Global Geodetic Observing System: Meeting the Requirements of a Global Society on a Changing Planet in 2020, Springer, ISBN-10: 3642026869, ISBN-13: 978-3642026867. http://dx.doi.org/10.1007/978-3-642-02687-4

[9] International GNSS Service webpage: http://igscb.jpl.nasa.gov/

[10] International Laser Ranging Service: http://ilrs.gsfc.nasa.gov/

[11] Wood R. and Sherwood R., 2001.IGSMAIL-3373: HERS antenna problem.

[12] Wood R. and Sherwood R., 2001.IGSMAIL-3503: HERS data flow restarted. 